\title{
Treponema endosymbionts are the dominant bacterial members with ureolytic potential in the gut of the wood-feeding termite, Reticulitermes hesperus
}

Jonathan Y Lin ( $\nabla$ johlin@ucdavis.edu )

University of California Davis https://orcid.org/0000-0003-4977-2506

Laibin Huang

University of California Davis

Sung $J$ Won

University of California Davis

Jorge L.M. Rodrigues

University of California Davis

\section{Research Article}

Keywords: Urease, ureC gene, gut microbiome, termite gut, endosymbiont, Treponema

Posted Date: March 28th, 2022

DOI: https://doi.org/10.21203/rs.3.rs-1225749/v2

License: (c) (1) This work is licensed under a Creative Commons Attribution 4.0 International License.

Read Full License 
Treponema endosymbionts are the dominant bacterial members with ureolytic potential in the gut of the wood-feeding termite, Reticulitermes hesperus

\title{
ORCID
}

Jonathan Y. Lin: 0000-0003-4977-2506

Laibin Huang: 0000-0003-3885-1815

Jorge L.M. Rodrigues: 0000-0002-6446-6462

Running Title: Microbial urease genes in termite guts

Keywords: Urease, ureC gene, gut microbiome, termite gut, endosymbiont, Treponema

1'Department of Land, Air, and Water Resources, University of California, Davis, CA, USA 95616 Laboratory, Berkeley, CA, USA 94720

${ }^{*}$ Correspondence: jmrodrigues@ucdavis.edu

\author{
Running Title: Microbial urease genes in termite guts
}


Abstract

Termites are remarkable for their ability to digest wood as their main energy source, but

51 the extremely low nitrogen $(\mathrm{N})$ content of their diet presents a major challenge for $\mathrm{N}$ acquisition.

52 Besides the $\mathrm{N}_{2}$-fixing bacteria in the gut, the symbiotic groups that recycle $\mathrm{N}$ from waste

53 products as a complementary $\mathrm{N}$-provisioning mechanism in termites remains poorly understood.

54 In this study, we used a combination of high-throughput amplicon sequencing, quantitative PCR,

55 and cultivation to characterize the microbial community capable of degrading urea, a common

56 waste product, into ammonia in the guts of two colonies of Reticulitermes hesperus termites.

57 The abundance of the ureC gene, which encodes for the alpha subunit of the urease enzyme,

58 ranged from 0.43 to $2.93 \%$ of the total prokaryotic community. Taxonomic analysis indicated

59 that $27.6 \%$ of the ureC gene amplicons in the termite gut matched with a Treponema

60 endosymbiont of gut protists previously found in several other termites, suggesting an important

61 contribution to the nutrition of essential cellulolytic protists. This corroborated our cultivation

62 efforts, where a majority of the isolates recovered had ureolytic potential and matched the other

63 taxa from our ureC gene sequences. Together, our results underscore a more important role for

64 ureolysis by endosymbionts within protists than by free-swimming bacteria in the gut lumen of

$65 \quad$ R. hesperus.

66

67

68

69

70

71

72

73 
Termites are social insects descended from wood-feeding cockroaches [1] and have long

75 been studied for their ability to digest lignocellulose [2, 3]. This ability is driven by essential 76 contributions from deeply evolved, mutualistic symbionts found in their hindguts consisting of

77 archaea and bacteria in the 'higher termites' (Family Termitidae) and a tripartite community of

78 free-swimming archaea, bacteria, and cellulolytic protists with their ecto- and endosymbionts in

79 the evolutionarily basal 'lower termites' [2, 3]. While biological nitrogen $\mathrm{N}_{2}$ fixation (BNF) by

80 bacterial symbionts is a prominent route of nitrogen $(\mathrm{N})$ acquisition in termites to compensate

81 for their N-limited diet [1-3], BNF rates have been reported to be highly variable, suggesting that

82 some species with low BNF rates such as the Reticulitermes must rely on different pathways to

83 satisfy all host nutrient requirements [4]. Termites release most nitrogenous waste as uric acid,

84 and a previous study showed that gut bacteria in wood-feeding Reticulitermes flavipes termites

85 are capable of recycling $\mathrm{N}$ in uric acid for re-absorption into host tissue [5]. This process was

86 confirmed in several bacterial strains isolated from termites that can ferment uric acid to produce

87 ammonia [6, 7]. However, uric acid can be converted into urea by enzymes produced by the host

88 or symbionts [8]; urea can be excreted as a waste product by protist cells [9]. At this step,

89 whether there are symbionts that can produce urease enzymes to catalyze the breakdown of

90 urea to ammonia for re-assimilation in the termite gut remains unknown. This represents a

91 knowledge gap in symbiont-mediated processes that may affect termite nutrition. To fill this gap,

92 we characterized the taxonomic diversity and abundance of symbionts with ureolytic potential

93 in the hindguts of two colonies of termites.

94 Termites were collected from two sites in the Central Valley in CA, USA (Fig. 1a).

95 Sequencing of the mitochondrial cytochrome oxidase II (COII) gene identified them as two

96 genetically distinct colonies of Reticulitermes hesperus (Fig. 1b). DNA was extracted from

97 hindguts dissected from workers and the ureC gene, which encodes for the alpha subunit of the

98 urease enzyme, was sequenced (Supplementary methods). The resulting sequences were 
99 classified using GraftM against a ureC gene reference package [10]. Quantitative PCR (qPCR)

100 was performed to determine the abundance of ure $C$ genes in the termite gut, and bacteria were

101 cultivated from hindgut homogenates and screened to confirm ureolytic potential. Across all

102 samples, the composition of ureC gene at the phylum level consisted of the Proteobacteria

103 (36.7\%), Spirochaetes (27.6\%), and Firmicutes (21.7\%), with the remaining sequences not

104 classified to any phyla (12.0\%). The most abundant ureC sequences classified at the genus level

105 were the Treponema (27.6\%), followed by Candidatus Accumulibacter (7.3\%), Pseudomonas

106 (4.2\%), Bacillus (3.0\%), Acinetobacter (1.8\%), and Desulfovibrio (1.1\%) (Fig. 1c). Notably, all ureC

107 gene sequences annotated as Treponema were mapped to a single phylotype, Urec_98, which

108 was the most abundant classified ure $C$ sequence for both colonies. Phylogenetic analysis placed

109 Urec_98 in a clade with a species previously identified as "Candidatus Treponema

110 intracellularis," an endosymbiont of Eucomonympha protists in the termite gut [11] (Fig. 2).

111 Urec_98 shared a $96.61 \%$ similarity in the protein coding sequence with the ureC gene from

112 these endosymbionts and was genetically divergent from the sequences of the free-living

113 Treponema bryantii and T. ruminis (71.97\% identity for both), indicating that Urec_98 is

114 evolutionarily distant from other Treponema species with ureolytic potential and likely an

115 endosymbiont of protists in the termite gut. The abundance of ure $C$ gene ranged from 131 to

1162,171 copies $n g \mathrm{DNA}^{-1}$, which was 0.43 to $2.93 \%$ of the total prokaryotic community when

117 calculated as a proportion of the 16S rRNA gene copies (Table 1). Out of a total of 192 isolates

118 retrieved from our cultivation procedures, most of the identified strains were from the phyla

119 Proteobacteria (148 isolates), Bacteroidetes (11 isolates) and Firmicutes (6 isolates) (Table S1).

120 Approximately $92.2 \%$ of these isolates possessed ureolytic potential based on diagnostic PCR

121 of the ureC gene, and the most abundant identified strains were from the genus Citrobacter (88

122 isolates), Pseudomonas (28 isolates), Acinetobacter (27 isolates), Chryseobacterium (11 isolates), 
123 and Bacillus (5 isolates) (Table S1). Taken together, our results demonstrate that the ureC gene

124 is present in the termite gut with a majority of the sequences represented by endosymbionts.

125 Urec_98 was the single most abundant phylotype from our ureC gene dataset and is

126 closely related to "Candidatus Treponema intracellularis," an endosymbiont of Eucomonympha

127 protists previously found in wood-feeding Hodotermopsis sjoestedti termites [11]. The

128 "Candidatus T. intracellularis" genome contains genes encoding for urease as well as a

129 membrane-bound urea channel, indicating its ability to both transport and use urea excreted by

130 its host [11]. "Candidatus T. intracellularis" falls within the termite Treponema cluster II [11], a

131 defined clade of Treponema ectosymbionts attached to the cell surface of termite gut protists

$132[12,13]$. This clade, along with a group of free-swimming Treponema (cluster I) comprise an

133 abundant and highly co-evolved community of Spirochaetes within the termite gut [14]. To date,

134 the only other members of the Treponema for which genomes are available on NCBI that possess

135 genes for urease and urea transporters are T. bryantii and T. ruminis, two Spirochaetes originally

136 isolated from the bovine rumen $[15,16]$, an environment where urea from the bloodstream is a

137 major source of waste $\mathrm{N}$ in ruminant animals [17]. By comparison, the genomes of T. primitia

138 and T. azonutricium [18], two free-swimming species from Treponema cluster I isolated from the

139 termite gut [19], do not contain genes encoding for any urease subunits or urea transporters.

140 This suggests that unlike the rumen environment, the termite gut lumen likely does not have a 141 significant flux of urea which may underscore a lack of selective pressure for free-swimming 142 Spirochaetes to possess urease genes.

143 Besides the Treponema endosymbionts, several extracellular bacteria previously isolated

144 from termite guts have been shown to have urease enzyme activity, such as Comamonas

145 odontotermitis [20]; or encode operons for urease and their transporters in their genomes

146 including a Citrobacter strain [21], Sporomusa termitida [22], Stenoxybacter acetivorans [23], and

147 several Verrucomicrobia strains [24-28]. Yet, the fact that we did not detect any of these taxa at 
148 proportions greater than $0.5 \%$ in our ureC gene dataset suggests that ureolysis in the gut lumen

149 by free-swimming bacteria likely does not produce a significant quantity of recycled $\mathrm{N}$ for

150 Reticulitermes termites. By contrast, our detection of Treponema endosymbionts at much higher

151 proportions from our ure $C$ sequences suggests a more important role for urea recycling inside

152 protists. This is substantiated by a previous finding of another termite endosymbiont,

153 "Candidatus Azobacteroides pseudotrichonymphae," a Bacteroidales strain that, like

154 "Candidatus T. intracellularis," also possesses a gene cluster encoding a urease and urea

155 transporter [29]. This endosymbiont was found to be abundant in Pseudotrichonympha protists,

156 a sister lineage to the Eucomonympha protists, which suggests that phylogenetically distant

157 bacteria may have convergently established similar functional niches for $\mathrm{N}$ recycling within

158 protist hosts [11]. These contributions are expected to enable the protists to grow efficiently and 159 remain stable during nutrient fluctuations in the gut, thereby allowing the termite to maintain 160 cellulolytic protists essential for host nutrition [11].

161 In summary, our characterization of the ureC gene in termite guts showed a significant

162 number of sequences matching to a Treponema endosymbiont of protists previously found in

163 another termite, highlighting a more important role for ureolysis by endosymbionts within protists

164 than by free-swimming bacteria in the gut lumen of $R$. hesperus. Thus, ureolytic endosymbionts

165 are likely important for maintaining the stability of essential cellulolytic protists within the tripartite

166 microbial community in the guts of the lower termites. Future work on ureolytic gut microbes is

167 needed to determine the distribution and ecological relevance of Treponema endosymbionts in 168 other termites. 


\section{Acknowledgements}

174 We thank the members of the Soil Ecogenomics lab, Eloi Parladé, Albert Barberán, and Michael

175 Messina for assistance with sampling. We acknowledge Yang Ouyang for uploading ureC seed

176 sequences to the Fungene Repository.

178 Declarations

179 Funding

180 This research was supported in part by a UC Davis Henry A. Jastro Research Award (to J.Y.L.)

181 and a UC Davis Provost's Undergraduate Fellowship (to S.J.W.).

183 Competing Interests

184 All authors declare that they have no competing interests.

\section{Ethics Approval}

187 No approval of research ethics committees was required to accomplish the goals of this study

188 because experimental work was conducted with an unregulated invertebrate species.

190 Data Availability

191 The ureC gene sequences were deposited to the NCBI sequence read archive (SRA) under

192 BioProject PRJNA660442 with the BioSample accession number SAMN15949813. The custom

193 GraftM gene package used for taxonomic classification of ureC gene sequences is available at

194 https://github.com/jonathanylin/Termite_gut_urease.

195

196 Author Contributions 
197 JYL and JLMR contributed to the study conception and design. Material preparation, data 198 collection, and analysis were performed by JYL, LH, and SJW. JYL wrote the manuscript and all 199 authors commented on previous versions of the manuscript. All authors read and approved the 200 final version of the manuscript.

201

202

203

204

205

206

207

208

209

210

211

212

213

214

215

216

217

218

219

220

221

222

223

224

225

226

227

228

229

230

231

232

233

234

235

236

237

238

239

240

241 


\section{References}

1. Brune A, Dietrich $C$ (2015) The gut microbiota of termites: Digesting the diversity in the light of ecology and evolution. Ann Rev Microbiol 69:145-166.

2. Wertz JT, Béchade B (2020) Symbiont-mediated degradation of dietary carbon sources in social herbivorous insects. In: Oliver KM, Russell JA (eds) Advances in Insect Physiology. Academic Press, pp 63-109

3. Brune A (2014) Symbiotic digestion of lignocellulose in termite guts. Nat Rev Microbiol 12:168-180. https://doi.org/10.1038/nrmicro3182

4. Breznak JA (2000) Ecology of prokaryotic microbes in the guts of wood- and litter-feeding termites. In: Abe T, Bignell DE, Higashi M (eds) Termites: Evolution, Sociality, Symbioses, Ecology. Springer Netherlands, Dordrecht, pp 209-231

5. Potrikus CJ, Breznak JA (1981) Gut bacteria recycle uric acid nitrogen in termites: A strategy for nutrient conservation. Proc Natl Acad Sci USA 78:4601-4605

6. Potrikus CJ, Breznak JA (1980) Anaerobic degradation of uric acid by gut bacteria of termites. Appl Environ Microbiol 40:125-132

7. Thong-On A, Suzuki K, Noda S, et al (2012) Isolation and characterization of anaerobic bacteria for symbiotic recycling of uric acid nitrogen in the gut of various termites. Microbes Environ 27:186-192

8. Hansen AK, Pers D, Russell JA (2020) Symbiotic solutions to nitrogen limitation and amino acid imbalance in insect diets. In: Oliver KM, Russell JA (eds) Advances in Insect Physiology. Academic Press, pp 161-205

9. Weatherby JH (1929) Excretion of nitrogenous substances in Protozoa. Physiol Zool 2:375-394. https://doi.org/10.1086/physzool.2.3.30151076

10. Boyd JA, Woodcroft BJ, Tyson GW (2018) GraftM: A tool for scalable, phylogenetically informed classification of genes within metagenomes. Nucleic Acids Res 46:e59-e59. https://doi.org/10.1093/nar/gky174

11. Ohkuma M, Noda S, Hattori S, et al (2015) Acetogenesis from $\mathrm{H}_{2}$ plus $\mathrm{CO}_{2}$ and nitrogen fixation by an endosymbiotic spirochete of a termite-gut cellulolytic protist. Proc Natl Acad Sci USA 112:10224-10230. https://doi.org/10.1073/pnas.1423979112

12. Ohkuma M, lida T, Kudo T (1999) Phylogenetic relationships of symbiotic Spirochetes in the gut of diverse termites. FEMS Microbiol Lett 181:123-129. https://doi.org/10.1111/j.1574-6968.1999.tb08834.x

13. Noda S, Ohkuma M, Yamada A, et al (2003) Phylogenetic position and in situ Identification of ectosymbiotic Spirochetes on protists in the termite gut. Appl Environ Microbiol 69:625-633. https://doi.org/10.1128/AEM.69.1.625-633.2003 
14. Brune A (2006) Symbiotic associations between termites and prokaryotes. In: Dr MDP, Falkow S, Rosenberg E, et al (eds) The Prokaryotes. Springer New York, pp 439-474

15. Stanton TB, Canale-Parola E (1980) Treponema bryantii sp. nov., a rumen spirochete that interacts with cellulolytic bacteria. Arch Microbiol 127:145-156.

16. Newbrook K, Staton GJ, Clegg SR, et al (2017) Treponema ruminis sp. nov., a spirochaete isolated from the bovine rumen. Int J Syst Evol Microbiol 67:1349-1354. https://doi.org/10.1099/ijsem.0.001812

17. Mobley HL, Hausinger RP (1989) Microbial ureases: Significance, regulation, and molecular characterization. Microbiol Rev 53:85-108

18. Graber JR, Leadbetter JR, Breznak JA (2004) Description of Treponema azotonutricium sp. nov. and Treponema primitia sp. nov., the first Spirochetes isolated from termite guts. Appl Environ Microbiol 70:1315-1320. https://doi.org/10.1128/AEM.70.3.1315-1320.2004

19. Leadbetter JR, Schmidt TM, Graber JR, Breznak JA (1999) Acetogenesis from $\mathrm{H}_{2}$ plus $\mathrm{CO}_{2}$ by Spirochetes from termite guts. Science 283:686-689. https://doi.org/10.1126/science.283.5402.686

20. Chou J-H, Sheu S-Y, Lin K-Y, et al (2007) Comamonas odontotermitis sp. nov., isolated from the gut of the termite Odontotermes formosanus. Int J Syst Evol Microbiol 57:887891. https://doi.org/10.1099/ijs.0.64551-0

21. Fontes-Perez H, Olvera-García M, Chávez-Martínez A, et al (2015) Genome sequence of Citrobacter sp. CtB7.12, isolated from the gut of the desert subterranean termite Heterotermes aureus. Genome Announc 3:. https://doi.org/10.1128/genomeA.01290-15

22. Breznak JA, Switzer JM, Seitz H-J (1988) Sporomusa termitida sp. nov., an $\mathrm{H}_{2} / \mathrm{CO}_{2}$ utilizing acetogen isolated from termites. Arch Microbiol 150:282-288. https://doi.org/10.1007/BF00407793

23. Wertz JT, Breznak JA (2007) Stenoxybacter acetivorans gen. nov., sp. nov., an acetateoxidizing obligate microaerophile among diverse $\mathrm{O}_{2}$-consuming bacteria from termite guts. Appl Environ Microbiol 73:6819-6828. https://doi.org/10.1128/AEM.00786-07

24. Wertz JT, Kim E, Breznak JA, et al (2012) Genomic and physiological characterization of the Verrucomicrobia isolate Diplosphaera colitermitum gen. nov., sp. nov., reveals microaerophily and nitrogen fixation genes. Appl Environ Microbiol 78:1544-1555. https://doi.org/10.1128/AEM.06466-11

25. Lin JY, Rodrigues JLM (2020) Geminisphaera. In: Bergey's Manual of Systematics of Archaea and Bacteria. Wiley, pp 1-7

26. Kotak M, Lin JY, Isanapong J, Rodrigues JLM (2020) Draft genome sequences of strains TAV3 and TAV4 (Verrucomicrobia: Opitutaceae), isolated from a wood-feeding termite, and in silico analysis of their polysaccharide-degrading enzymes. Microbiol Resour Announc 9: https://doi.org/10.1128/MRA.01192-19 
27. Kotak M, Isanapong J, Goodwin L, et al (2015) Complete genome sequence of the Opitutaceae bacterium strain TAV5, a potential facultative methylotroph of the woodfeeding termite Reticulitermes flavipes. Genome Announc 3: https://doi.org/10.1128/genomeA.00060-15

28. Isanapong J, Goodwin L, Bruce D, et al (2012) High-quality draft genome sequence of the Opitutaceae bacterium strain TAV1, a symbiont of the wood-feeding termite Reticulitermes flavipes. J Bacteriol 194:2744-2745. https://doi.org/10.1128/JB.00264-12

29. Hongoh $\mathrm{Y}$, Sharma VK, Prakash T, et al (2008) Genome of an endosymbiont coupling $\mathrm{N}_{2}$ fixation to cellulolysis within protist cells in termite gut. Science 322:1108-1109. https://doi.org/10.1126/science.1165578

\section{Tables}

Table 1. Abundance and proportion of ure $C$ genes in termite guts.

\begin{tabular}{|c|c|c|c|c|c|}
\hline Sample & Colony & ure $C$ copies ng DNA ${ }^{-1}$ & 16S rRNA copies ng DNA ${ }^{-1}$ & ure $C \mid 16 S$ copies & ure C relative abundance (\%) \\
\hline LT1 & $\mathrm{TH} 1$ & 310 & 40678 & 0.0076 & 0.76 \\
\hline LT2 & TH1 & 371 & 59087 & 0.0063 & 0.63 \\
\hline LT3 & TH1 & 648 & 67205 & 0.0096 & 0.96 \\
\hline LT4 & TH1 & 910 & 103702 & 0.0088 & 0.88 \\
\hline LT5 & TH1 & 178 & 41056 & 0.0043 & 0.43 \\
\hline LT6 & TH1 & 437 & 64671 & 0.0068 & 0.68 \\
\hline LT7 & TH1 & 851 & 78132 & 0.0109 & 1.09 \\
\hline LT8 & TH1 & 439 & 32391 & 0.0136 & 1.36 \\
\hline LT9 & TH1 & 361 & 54237 & 0.0067 & 0.67 \\
\hline LT10 & $\mathrm{TH} 1$ & 413 & 67608 & 0.0061 & 0.61 \\
\hline LT11 & TH1 & 131 & 30121 & 0.0044 & 0.44 \\
\hline LT12 & $\mathrm{TH} 1$ & 653 & 87496 & 0.0075 & 0.75 \\
\hline WT1 & WTH1 & 1872 & 127482 & 0.0147 & 1.47 \\
\hline WT2 & WTH1 & 833 & 54301 & 0.0153 & 1.53 \\
\hline WT3 & WTH1 & 502 & 43315 & 0.0116 & 1.16 \\
\hline WT4 & WTH1 & 1033 & 42190 & 0.0245 & 2.45 \\
\hline WT5 & WTH1 & 1153 & 39813 & 0.0290 & 2.90 \\
\hline WT6 & WTH1 & 2171 & 84579 & 0.0257 & 2.57 \\
\hline WT7 & WTH1 & 995 & 43408 & 0.0229 & 2.29 \\
\hline WT8 & WTH1 & 724 & 50891 & 0.0142 & 1.42 \\
\hline WT9 & WTH1 & 1413 & 56497 & 0.0250 & 2.50 \\
\hline WT10 & WTH1 & 1486 & 76989 & 0.0193 & 1.93 \\
\hline WT11 & WTH1 & 1314 & 44906 & 0.0293 & 2.93 \\
\hline
\end{tabular}


357 Fig. 1. a) Map showing the collection sites for the termite colonies used in this study. b)

358 Phylogenetic tree (maximum-likelihood) based on termite mitochondrial cytochrome oxidase II 359 (COII) gene sequences showing the identification of the termite colonies. Colors correspond

360 with sampling locations, numbers at branch nodes indicate bootstrap support (500 replicates)

361 above $50 \%$, and the bar shows 0.1 substitutions per position. c) Alluvial plot showing the

362 distribution of classified gut ureolytic microbial communities based on the ure $C$ gene in all

363 termites across different taxonomic ranks. The relative abundance (\%) for the taxa at the genus

364 level are shown in parentheses.

365

366

367

Fig. 2. Phylogenetic characterization of Urec_98 based on protein-coding sequences of the ure $C$ gene. Sequences from other Treponema species, bacteria previously isolated from termite guts, and other strains spanning major bacterial phyla for which data are available were included as reference species. The tree was constructed by the maximum-likelihood method using the Jones-Thorton-Taylor model. Numbers at branch nodes indicate bootstrap support (500 replicates) above $50 \%$, sequence accession numbers are given in parentheses, and the bar shows 0.1 substitutions per position. 


\section{Figures}

\section{Figure 1}

a) Map showing the collection sites for the termite colonies used in this study. b) Phylogenetic tree (maximum-likelihood) based on termite mitochondrial cytochrome oxidase II (COII) gene sequences showing the identification of the termite colonies. Colors correspond with sampling locations, numbers at branch nodes indicate bootstrap support (500 replicates) above $50 \%$, and the bar shows 0.1 substitutions per position. c) Alluvial plot showing the distribution of classified gut ureolytic microbial communities based on the ureC gene in all termites across different taxonomic ranks. The relative abundance (\%) for the taxa at the genus level are shown in parentheses.

\section{Figure 2}

Phylogenetic characterization of Urec_98 based on protein-coding sequences of the ureC gene. Sequences from other Treponema species, bacteria previously isolated from termite guts, and other strains spanning major bacterial phyla for which data are available were included as reference species. The tree was constructed by the maximum-likelihood method using the Jones-Thorton-Taylor model. Numbers at branch nodes indicate bootstrap support (500 replicates) above $50 \%$, sequence accession numbers are given in parentheses, and the bar shows 0.1 substitutions per position.

\section{Supplementary Files}

This is a list of supplementary files associated with this preprint. Click to download.

- TermiteUreaseManuscriptSupplementREVISEDFINAL.pdf 